\title{
Volumetric Overload Shocks: Why Is Starling's Law for Capillary Interstitial Fluid Transfer Wrong? The Hydrodynamics of a Porous Orifice Tube as Alternative
}

\author{
Ahmed N. Ghanem1, Salma A. Ghanem² \\ ${ }^{1}$ Retired Consultant Urologist, Mansoura, Egypt \\ ${ }^{2}$ Guys \& St. Thomas NHS Trust, London, UK \\ Email:an_ghanem@hotmail.com,salma.ghanem@gstt.nhs.uk, Salmaliciouss@hotmail.com
}

Received 27 April 2016; accepted 31 May 2016; published 3 June 2016

Copyright (C) 2016 by authors and Scientific Research Publishing Inc. This work is licensed under the Creative Commons Attribution International License (CC BY). http://creativecommons.org/licenses/by/4.0/

(c) () Op Open Access

\section{Abstract}

Based on clinical and experimental work two new types of volumetric overload shocks are reported: volumetric overload shock type one and type two depending on the type of fluid causing their induction. Volumetric overload shock type one is induced by sodium-free fluids such as glycine, glucose, mannitol and sorbitol and is characterized with acute dilutional hyponatraemia. Volumetric overload shock type 2 is induced with sodium-based fluids normal saline and plasma substitutes used for resuscitation of the critically ill and has no serological marker. It presents with the multiple vital organs dysfunction or failure syndrome or the adult respiratory distress syndrome. Hypertonic sodium is an effective treatment when given early adequately. The underlying pathophysiology is discussed. An alternative to Starling's law for the capillary interstitial fluid transfer is given. Hydrodynamic of a porous orifice tube akin to capillary with a surrounding Chamber akin to the interstitial fluid space demonstrated a rapid dynamic magnetic field-like fluid circulation between the surrounding chamber and the lumen of the $G$ tube that represent an adequate replacement for Starling's law.

\section{Keywords}

Shock, The Multiple Vital Organs Dysfunction or Failure Syndrome, The Adult Respiratory Distress Syndrome, The Transurethral Resection of the Prostate Syndrome, Hyponatraemia 


\section{Introduction}

Volumetric Overload Shock (VOS) is a condition caused by massive fluid infusions and is of two types: Type one (VOS1) and Type two (VOS2). VOS1 is induced by sodium-free fluid gain such as 1.5\% Glycine used as irrigant during the Transurethral Resection of the Prostate (TURP) [1]. It has been reported with other irrigants such as Glucose, Mannitol and Sorbitol. It is known as the TURP syndrome or hyponatraemic shock [2] as Hyponatraemia (HN) is a marked serological marker for the condition. VOS2 is induced by massive infusion of sodium-based fluids such as normal saline, Ringer, Hartmann, plasma and plasma substitutes and/or blood transfusions that may complicate the therapy of VOS1. VOS2 also complicates fluid therapy in critically ill patients suffering from other known shocks such as hypovolaemic, haemorrhagic and septicaemic and presents with the Multiple Vital Organs Dysfunction (MVOD) or failure syndrome. Adult Respiratory Distress Syndrome (ARDS) [3] is another name under which VOS2 is reported. The occurrence of massive interstitial tissue oedema with congestion of vital organs, pleural and peritoneal effusion, in the presence of severe hypotension shock, casted doubt on Starling's law!

\section{Aetiology}

Both conditions of VOS1 and VOS2 are caused by volumetic overload. VOS1 is induced by the infusion of 3.5 litres of Glycine irrigant through the periprostatic veins [1]. Intravenous infusions of 5\% Glucose augment this effect. The gain of 3.5 - 5 litres induces typical VOS1. It is important to realize the significance of time; 3.5 litres of fluids is a normal daily intake if gained over 24 hours but is certainly pathological if gained over one hour. VOS2 is induced by the gain of 12 - 14 litres of sodium-based fluids when reported in ARDS [3]. The problem here is that every hypotension is considered synonimus with hypovolaemia, and is treated with massive volume expansion. In the past VOS1 was wrongly attributed to haemorrhagic, hypovolaemic or cardiogenic shock hence treated with sodium-based fluids and VOS2 was induced [4].

\section{Pathophysiology}

Shock is a disturbance at the capillary cellular level impairing the capillary-interstitial fluid transfer; delivery of oxygen and removal of waste products. The process is governed by Starling's law [5]. In this law the arterial pressure is considered the force causing capillary filtration! If this is true, how come that arterial hypertension though common never causes oedema? Starling based his hypothesis on Poiseuille work on strait uniform brass tubes. Latter evidence however demonstrated that the capillary is a porous narrow orifice $(G)$ tube as it has a pre-capillary sphincter [6] and pores that allow the passage of plasma proteins [7]. Because the capillary pores allow the passage of plasma molecules a call for reconsideration of Starling's hypothesis was made [8].

The hydrodynamics of the $G$ tube demonstrated that the proximal (arterial) pressure induces a negative side pressure gradient on the wall of the $G$ tube causing suction most prominent over the proximal half and turns into positive pressure over the distal half [9] (Figure 1). Incorporating the G tube in a chamber (C), representing the

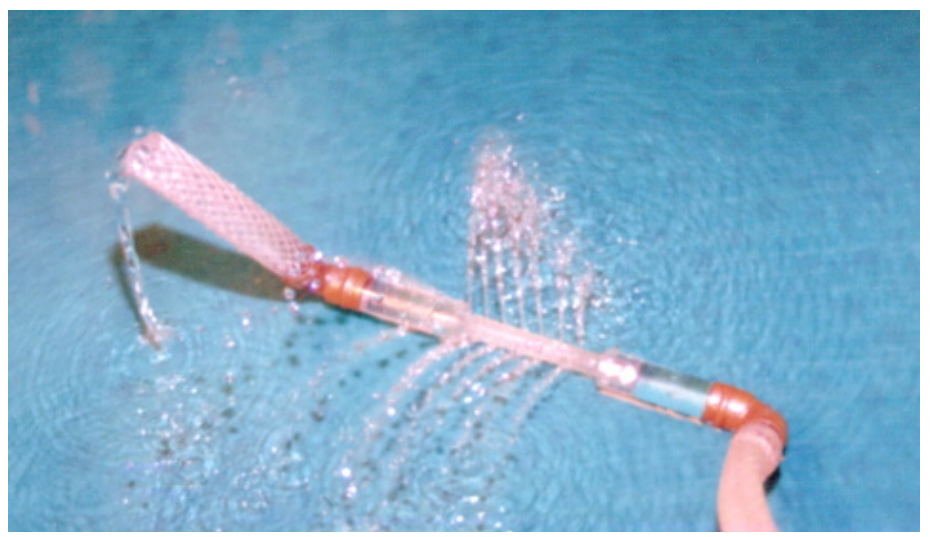

Figure 1. Shows the hydrodynamics of the porous orifice (G) tube. There is a pressure gradient at the wall of the $G$ tube starting at the borifice with high suction and ending at exit with high filtration. The water comes out over the distal part and is sucked in over the proximal part of the $\mathrm{G}$ tube. 
interstitial space surrounding a capillary, demonstrated a rapid dynamic magnetic field-like fluid circulation between the $\mathrm{C}$ and $\mathrm{G}$ tube lumen (Figure 2). Incorporating the $\mathrm{G}$ tube and $\mathrm{C}$ in a circulatory model driven by electric pump inducing proximal pressure similar to arterial pressure in human circulatory system; causing suction from $C$ into the lumen of $G$ tube. The pressure in $C$ is negative (Figure 3). The pressure in the interstitial fluid space is also negative [10]. The distal (venous) pressure is responsible for filtration. This proves that the circulatory system is not an all positive pressure, the arterial pressure causes suction not filtration at the capillary interstitial fluid circulation, and hence Starling’s law is wrong [9].

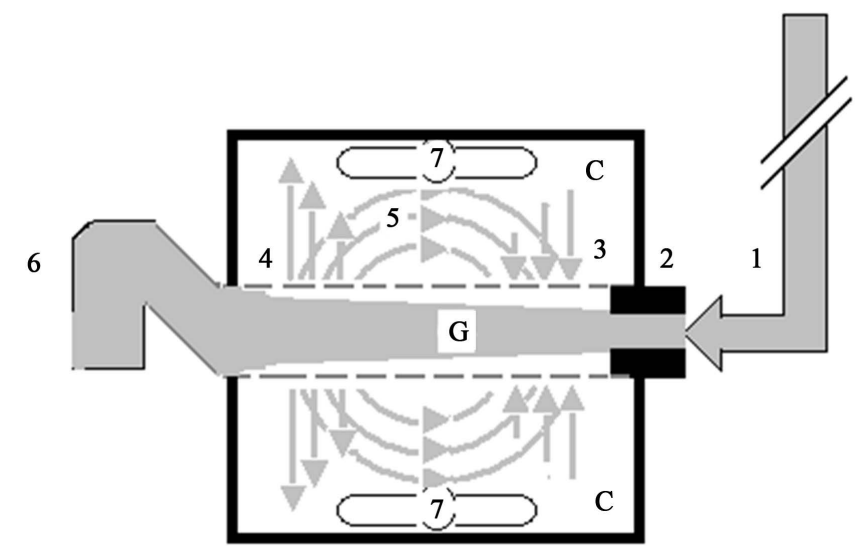

Figure 2. Shows diagram of the porous orifice (G) tube enclosed in chamber (C) demonstrating the magnetic field-like G-C circulation phenomenon. The proximal inflow (arterial) pressure (1) pushes fluid through the orifice (2) creating fluid jet in the lumen of the $G$ tube. The fluid jet creates negative side pressure gradient causing suction maximal over the proximal half of the $G$ tube near the inlet (3) that sucks fluid into lumen. The side pressure gradient turns positive pushing fluid out of lumen over the distal half maximally near the outlet (4). Thus the fluid around G tube inside C moves in magnetic field-like fluid circulation (5) taking an opposite direction to lumen flow of G. tube. The inflow (arterial) pressure 1 and orifice 2 induce the negative side pressure energy creating the dynamic G-C circulation phenomenon that is rapid, autonomous and efficient in moving fluid out from the $G$ tube lumen at 4, irrigating $C$ at 5 , then sucking it back again at 3, maintaining net negative energy pressure (7) inside C. The distal out flow (venous) pressure (6) enhances out flow at (4) and its elevation may turn the negative energy pressure 7 inside $C$ into positive, increasing volume and pressure inside $\mathrm{C}$ chamber.

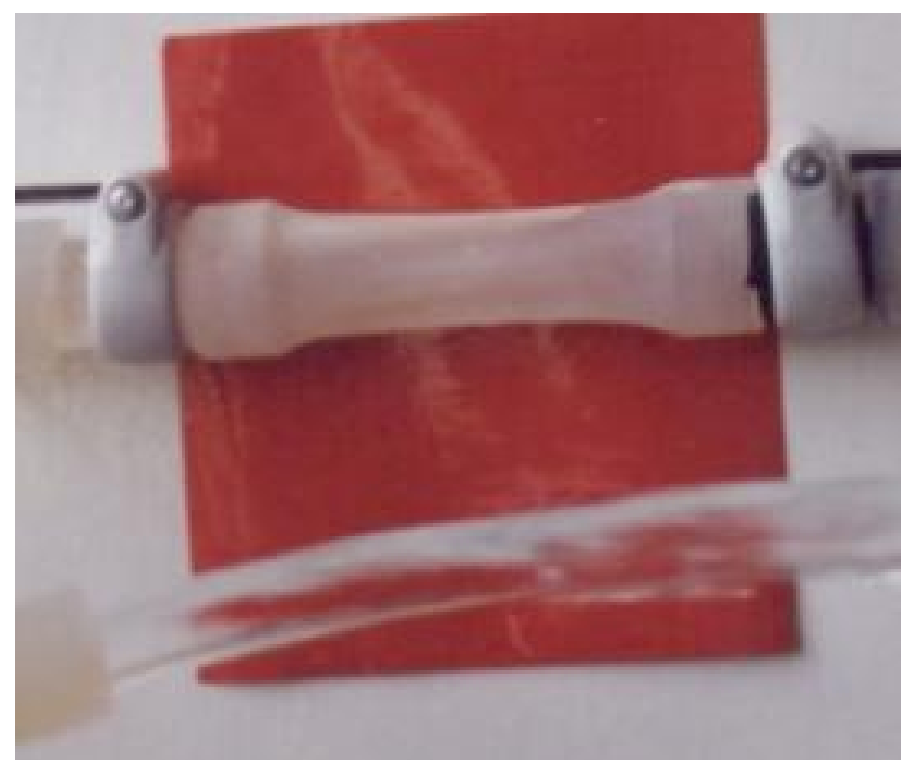

Figure 3. The chamber $\mathrm{C}$ around the $\mathrm{G}$ tube is made of soft rubber which is sucked in by the negative pressure gradient of the $\mathrm{G}$ tube. The interstitial pressure fluid has negative pressure of $-7 \mathrm{ml}$ water. 


\section{Serum Markers}

Dilutional HN is a clear serological marker of VOS1. The incidence and severity of HN of the TURP syndrome are reported [1] [4]. HN of $130 \mathrm{mmol} / \mathrm{l}$ is transient and self correcting, down to 125 is mild, further down to 120 is moderate, further drop is severe and manifests with a full blown condition of VOS1. A drop of serum sodium to a $100 \mathrm{mmol} / \mathrm{l}$ is usually lethal [4]. Unfortunately VOS2 has no clear serum marker. The only method of detection is the increase in body weight.

\section{Clinical Picture}

VOS1 has the following clinical picture before it transfers into VOS2 with a full blown picture of MVOD or failure or ARDS characterising both conditions. It is noted that VOS1 presents during surgery as hypotension shock and next day as hyponatraemic coma.

Cerebro-nervous system: Numbness and tingling sensation, sudden bilateral blindness and clouding of consciousness have been reported under spinal or epidural anaesthesia. Convulsion may occur. The patient proceeds into high scale coma and does not recover from general anaesthetic.

Cardiovascular system: Hypotension and bradycardia are early features also in patients having general anaesthetic. Hypertension is rarely detected. Other types of disrythmia with cardiac arrest and sudden death may occur. Cardiac enzymes are elevated. Cardiovascular shock prevails.

Respiratory system: The lungs are involved with shock lung or ARDS.

Renal: The kidneys develop annuria which is unresponsive to diuretics. Acute renal failure occurs. Serum urea and creatinine rise.

Hepatic system: The liver function tests are elevated.

General: Trunk oedema or anasarca develope.

The prognosis of VOS1 and VOS2 is bad; both end up in death when incorrectly diagnosed and inappropriately treated. With the use of treatment reported below the prognosis should tremendously improve.

\section{Treatment}

VOS1 is treated with hypertonic sodium solutions in the form of 5\% Sodium chloride and if not available $8.4 \%$ Sodium bicarbonate is equally effective. This is given in fractionated doses of $200 \mathrm{ml}$ over 10 minutes through a central venous line. The dose may be repeated up to 5 times. The effect of this therapy is magical [1] [4] [11]. The cardiovascular shock is corrected with elevation of arterial pressure. The patient recovers from coma. The kidneys respond with massive dieresis that should not be replaced. The treatment has proved equally effective in VOS2 that complicates VOS1 particularly when given early before the vital organs congestion develops into areas of necrosis and infarctions, and before MVOD becomes failure [4] [11]. Supportive measures on intensive care units are most helpful. If the kidneys do not respond by diuresis try haemodialysis—setting the net fluid balance to negative. Both VOS1 and VOS2 were induced in animals and treated successfully with hypertonic sodium [12].

\section{Conflict of Interest}

The author declares none.

\section{References}

[1] Ghanem, A.N. and Ward, J.P. (1990) Osmotic and Metabolic Sequelae of Volumetric Overload in Relation to the TURP Syndrome. British Journal of Urology, 66, 71-78. http://dx.doi.org/10.1111/j.1464-410X.1990.tb14868.x

[2] Harrison, R.H., Boren, J.S. and Robinson, J.R. (1956) Dilutional Hyponatraeic Shock Another Concept of the Transurethral Prostatic Reaction. Journal of Urology, 75, 95-110.

[3] Ashbaugh, D.G., Bigelow, D.B., Petty, T.L. and Levine, B.E. (1967) Acute Respiratory Distress in Adults. Lancet, 2, 319-323. http://dx.doi.org/10.1016/S0140-6736(67)90168-7

[4] Ghanem, A.N. (1988) The Transurethral Prostatectomy (TURP) Syndrome: An Investigation of the Osmotic and Metabolic Sequelae of Volumetric Overload (VO). MD Thesis, Institute of Urology \& Nephrology, Mansoura University, Egypt.

[5] Starling, E.H. (1886) Factors Involved in the Causation of Dropsy. Lancet, 2, 1266-1270, 1330-1334, 1406-1410. 
[6] Rhodin, J.A. (1967) The Ultra Structure of Mammalian Arterioles and Precapillary Sphincters. Journal of Ultrastructure Research, 18, 181-222. http://dx.doi.org/10.1016/S0022-5320(67)80239-9

[7] Karnovesky, M.J. (1967) The Ultra Structural Basis of Capillary Permeability Studied with Peroxidase as a Tracer. Journal of Cell Biology, 35, 213-236. http://dx.doi.org/10.1083/jcb.35.1.213

[8] Renkin, E.M. (1986) Some Consequences of Capillary Permeability to Macromolecules: Starling’s Hypothesis Reconsidered. American Journal of Physiology, 250, H706-H710.

[9] Ghanem, A.N. (2001) Magnetic Field-Like Fluid Circulation of a Porous Orifice Tube and Relevance to the CapillaryInterstitial Fluid Circulation: Preliminary Report. Medical Hypotheses, 56, 325-334. http://dx.doi.org/10.1054/mehy.2000.1149

[10] Guyton, A.C. and Coleman, T.G. (1968) Regulation of Interstitial Fluid Volume and Pressure. Annals of the New York Academy of Sciences, 150, 537-547. http://dx.doi.org/10.1111/j.1749-6632.1968.tb14705.x

[11] Ghanem, A.N., Wojtulewski, J.A. and Penney, M.D. (1987) Dangers in Treating Hyponatraemia. British Medical Journal, 294, 837. http://dx.doi.org/10.1136/bmj.294.6575.837-a

[12] Danowski, T.S., Winkler, A.W. and Elkington, J.R. (1946) The Treatment of Shock Due to Salt Depression; Comparison of Isotonic, of Hypertonic Saline and of Isotonic Glucose Solutions. Journal of Clinical Investigation, 25, 130. http://dx.doi.org/10.1172/JCI101682

\section{Abbreviations}

ARDS: $\quad$ The adult respiratory distress syndrome

VOS: $\quad$ Volumetric overload shock

VOS1: Volumetric overload shock type one

VOS2: $\quad$ Volumetric overload shock type 2

HN: Hyponatraemia

TURS: $\quad$ The transurethral resection of the prostate syndrome

MVOD: The multiple vital organs dysfunction or failure syndrome

G Tube: The porous narrow orifice tube 